\title{
CERTAIN CHARACTERIZATION OF SINGULAR SEMIGROUPS
}

\author{
BY TAKAYUKI TAMURA ${ }^{1}$
}

Communicated by Edwin Hewitt, February 20, 1962

1. According to Bruck [1], a groupoid means a system in which a binary operation is defined for any pair of elements. Consider groupoids satisfying one or two of the following conditions: (1) Any transformation is an endomorphism. (2) Any partition forms a factor groupoid. (3) Any subset is a subgroupoid. There actually exist such groupoids, for instance, singular semigroups defined as follows: By a right (left) singular semigroup we mean a groupoid defined by $x y=y(x y=x)$ for every $x, y$; and a singular semigroup shall stand for either a right singular or a left singular semigroup. In this note we shall report the results to relate what groupoids each of the above conditions or a somewhat weaker condition determines.

2. Let $S$ be a groupoid and let $a$ and $b$ be two distinct elements of $S$. For an ordered pair of $a$ and $b$, a transformation $\phi_{a, b}$ of $S$ is defined as follows:

$$
\phi_{a, b}(a)=b, \quad \phi_{a, b}(x)=x, \quad x \neq a .
$$

THEOREM 1. Every $\phi_{a, b}$ is an endomorphism of a groupoid $S$ if and only if $S$ is either a singular semigroup or a chain of order 2.

Since a chain of order 2 does not satisfy (1), we can easily have

CoRollary 1. Every transformation of a groupoid $S$ is an endomorphism of $S$ if and only if $S$ is a singular semigroup.

Denote by $f_{x, y}$ a substitution or a permutation which exchanges $x$ for $y$.

THEOREM 2. Every permutation of a groupoid $S$ is an automorphism of $S$ if and only if $S$ has one of the following types:

singular semigroups,

the groupoids $\{a, b\}$ of order 2:

\begin{tabular}{l|lll|ll}
$a$ & $a$ & $b$ & & $a$ & $b$ \\
$b$ & $b$ & $a$ & $a$ & $b$ & $b$ \\
$b$ & $b$ & $b$ & $a$ & $a$
\end{tabular}

1 This is a brief summary of a paper to be published elsewhere and so the precise proof will be given there. 
the idempotent quasigroup of order 3 (with unique type).

It is interesting that the transformations $\phi_{a, b}$ or permutations characterize singular semigroups in the range of groupoids with a few exceptional cases.

3. It has already been proved in [2] that any partition of a semigroup $S$ of order $\geqq 3$ is a decomposition of $S$ (i.e., a partition forming a factor groupoid), if and only if $S$ is either a singular semigroup or a zero semigroup defined as $x y=0$ for every $x, y$. However, it holds even if $S$ is a groupoid under a weaker condition. Let $x$ be an element of a groupoid $S$ and consider a partition of $S$ into the two classes: $S=\{x\} \cup(S-\{x\})$ where $\{x\}$ is the set of $x$ alone, and $S-\{x\}$ is its complement. This partition is denoted by $\Delta_{x}$.

THEOREM 3. Every $\Delta_{x}$ of a groupoid $S$ is a decomposition if and only if $S$ has one of the following types:

$(\alpha)$ singular semigroups,

$(\beta)$ zero semigroups: $x y=0$ for every $x, y$,

$(\gamma)$ the groupoids of order 2 besides the types $(\alpha),(\beta)$.

(There are 6 types in $(\gamma)$.)

COROLlARY 2. Every partition of a groupoid $S$ is a decomposition if and only if we have the same results as Theorem 3.

CoROLlaRy 3. If every partition of a groupoid $S$ is a decomposition of $S$, then every subgroupoid is a homomorphic image of $S$. However the converse is not true.

4. There are many types of groupoids any subset of which is a subgroupoid, so that no simple result can be derived, even in the range of semigroups.

Accordingly we shall try to find the types under stronger conditions. First, as the corollary of Theorem 3.

COROLlaRY 4. Every partition of a groupoid $S$ is a decomposition and every subset of $S$ is a subgroupoid if and only if $S$ is either a singular semigroup or a chain of order 2 .

Finally we have

THEOREM 4. Every subset of a groupoid $S$ is a subgroupoid which is a homomorphic image of $S$ if and only if $S$ is either a singular semigroup or a chain.

5. As the reader sees, only the condition of Corollary 1 characterizes a singular semigroup, but we have the following results with restriction. 
Corollary 5. A semigroup $S$ is a singular semigroup if and only if every permutation of $S$ is an automorphism.

Corollary 6. A groupoid $S$ of order $\geqq 3$ is a singular semigroup if and only if every $\phi_{a, b}$ is an endomorphism. This condition is equivalent to the following: every partition of a groupoid $S$ is a decomposition and every subset of $S$ is a subgroupoid.

COROLlary 7. A semigroup $S$ is a singular semigroup if and only if every subset is a subsemigroup of $S$, and $S$ is s-indecomposable. (That is, the greatest semilattice-homomorphic image of $S$ is one-element semigroup.)

\section{REFERENCES}

1. R. H. Bruck, A survey of binary systems, Springer-Verlag, Berlin, 1958.

2. M. Sasaki, Semigroups in which any partition is decomposition, The Annual Report of the Gakugei Faculty of the Iwate University, 19 (1961), 7-9.

TOKUSHIMa UNIVERSITY AND

University of California, Davis 